\title{
Custom Coordination Environments for Lanthanoids: Tripodal Ligands Achieve Near-Perfect Octahedral Coordination for Two ${ }_{3}$ Dysprosium-Based Molecular Nanomagnets
}

${ }_{4}$ Kwang Soo Lim, ${ }^{\dagger, \S}$ José J. Baldoví, ${ }^{\dagger, \S}$ ShangDa Jiang, ${ }^{\perp, \|, \S @ ~ B o n g ~ H o ~ K o o, ~}{ }^{\dagger}$ Dong Won Kang, ${ }^{\dagger}$ ${ }_{5}$ Woo Ram Lee, ${ }^{\dagger}$ Eui Kwan Koh, ${ }^{\text {, }}$ Alejandro Gaita-Ariño, ${ }^{*}, \odot$ Eugenio Coronado, ${ }^{+}$Michael Slota, ${ }^{\perp}$ 6 Lapo Bogani, ${ }^{*, \perp}$ and Chang Seop Hong ${ }^{*}, \odot$

$7^{\dagger}$ Department of Chemistry, Korea University, Seoul 136-713, Republic of Korea

8 "Instituto de Ciencia Molecular, Universidad de Valencia, C/Catedrático José Beltrán 2, Paterna E-46980, Spain

$9{ }^{\perp}$ Department of Materials, University of Oxford, 16 Parks Road, Oxford OX1 3PH, U.K.

10 "Inorganic Chemistry, College of Chemistry and Molecular Engineering, Peking University, Beijing 100871, P. R. China

${ }_{11}{ }^{\mathbb{T}}$ Nano-Bio System Research Team, Korea Basic Science Institute, Seoul 136-713, Korea

12 S Supporting Information

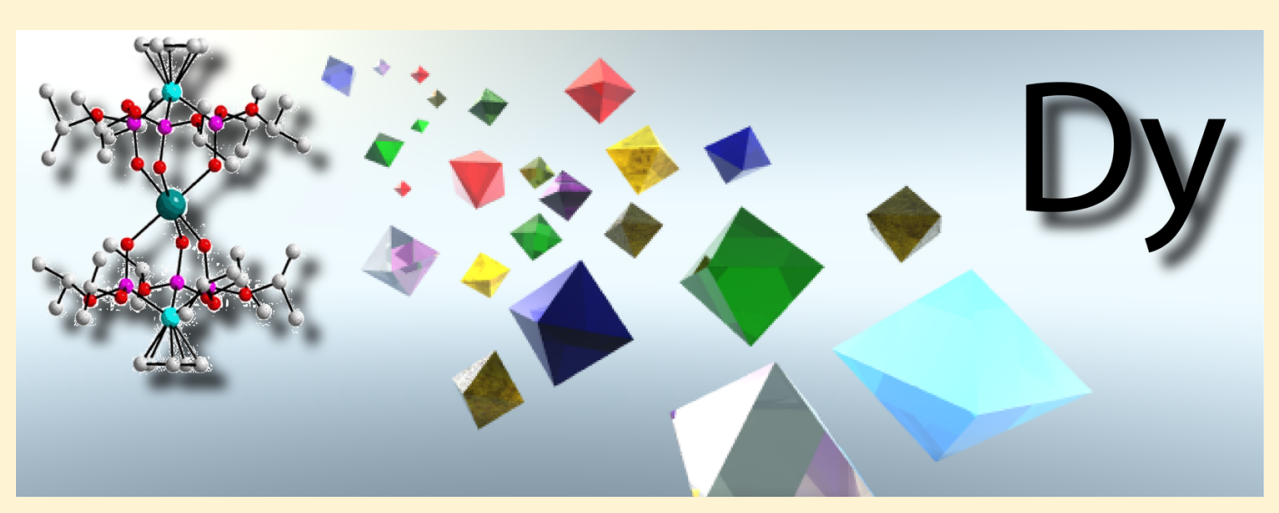

13 ABSTRACT: Controlling the coordination sphere of lanthanoid complexes is a challenging critical step toward controlling their 14 relaxation properties. Here we present the synthesis of hexacoordinated dysprosium single-molecule magnets, where tripodal 15 ligands achieve a near-perfect octahedral coordination. We perform a complete experimental and theoretical investigation of their 16 magnetic properties, including a full single-crystal magnetic anisotropy analysis. The combination of electrostatic and crystal-field 17 computational tools (SIMPRE and CONDON codes) allows us to explain the static behavior of these systems in detail.

\section{$18 \square$ INTRODUCTION}

19 Since the first observation of slow magnetic relaxation in 20 mononuclear lanthanide complexes $\left[\mathrm{Pc}_{2} \mathrm{Ln}\right]^{-}(\mathrm{Pc}=$ phthalo21 cyanide; $\left.\mathrm{Ln}^{\mathrm{III}}=\mathrm{Tb}, \mathrm{Dy}, \mathrm{Ho}\right){ }^{1}$ the field of mononuclear single22 ion magnets (SIMs) and molecular spin qubits has expanded 23 significantly and now includes multiple examples based on 24 either lanthanoids or transition metals, or even actinoids. ${ }^{2}$ 25 Considered to be classical bistable systems, they have set record 26 values for the temperatures at which slow relaxation of the 27 magnetization sets in. More interestingly, their quantum 28 properties make them very attractive as spin qubit systems. ${ }^{3}$ 29 Recently, record relaxation times have been obtained in 30 transition-metal complexes, where there is a greater control 31 over the coordination sphere. ${ }^{4}$ Moreover, in the quest for 32 longer relaxation times at higher temperatures, different authors 33 are now insisting on the need for exerting some control over 34 the molecular vibrations and not only focusing on the thermal 35 barrier. $^{2 b, 5}$ The physics behind these arguments shares common ground for both transition-metal and lanthanoid complexes, but 36 chemical control of the coordination sphere is different. This is 37 primarily due to the differences between the $\mathrm{d}$ and $\mathrm{f}$ orbitals 38 (the latter being more internal) and secondarily due to the 39 larger coordination spheres and higher coordination numbers 40 of the lanthanoids. Attempts to address this problem have been 41 performed by the use of rigid and bulky ligands such as 42 polyoxometalates. ${ }^{6}$ Still, full validation of the theoretical models 43 and the creation of a common background that can relate the 44 rare-earth magnetism to the ligand-field analysis are largely 45 unexplored. $^{\text {? }}$

Dysprosium(III), in particular, has seen much use in the 47 design of single-molecule magnets (SMMs) because of its high 48 magnetic anisotropy. ${ }^{8}$ In sandwich-type structures, dysprosium- 49 (III) complexes should theoretically display strong axial 50

Received: December 21, 2016 
51 anisotropy along the sandwich axis, but experiments show 52 substantial deviations from these predictions. Despite insight 53 obtained from octacoordinated dysprosium complexes with 54 square-antiprismatic geometries, where the properties can be 55 adjusted by local symmetry distortions and ligand substitution, ${ }^{9}$ 56 the lack of model systems and of treatable situations is 57 substantial. It has also been noted that special symmetries such 58 as the octahedron or cube can give rise to unique quantum 59 properties. As discussed previously by Baldovi et al., ${ }^{10}$ the 60 abundance of degenerate or near-degenerate electronic spin 61 energy levels, even prior to the consideration of nuclear spins, 62 creates a wealth of opportunities for avoided crossings and 63 therefore for clock transitions (CTs), which can be used for 64 improved quantum coherence, as we illustrated recently. ${ }^{11}$ 65 Furthermore, even in the absence of CTs, a sufficiently large 66 number of spin states in an accessible energy window can be 67 used to construct multiqubit (or d-bit) systems, where coherent 68 transitions are demonstrated. ${ }^{12}$

69 Compared to dysprosium-based magnetic systems with 70 coordination numbers larger than 7 , a hexacoordinated 71 dysprosium complex (not cluster) is rarely known ${ }^{13,14}$ and 72 highly sought after to further understand fundamental magnet73 like behaviors. Here we present the synthesis, structure, and 74 magnetic properties of Dy SMMs in custom-shaped and 75 uncommon octahedral coordination, providing model com76 pounds for the understanding of rare-earth magnetism.

\section{MATERIALS AND METHODS}

78 Reagents. All chemicals and solvents in the synthesis were of 79 reagent-grade and were used as received. $\mathrm{Na}\left[\mathrm{CpCo}\left\{\mathrm{P}(\mathrm{O})(\mathrm{OEt})_{2}\right\}_{3}\right]$ $80\left(\mathrm{NaL}_{\mathrm{OEt}}\right)$ and $\mathrm{Na}\left[\mathrm{CpCo}\left\{\mathrm{P}(\mathrm{O})(\mathrm{OiPr})_{2}\right\}_{3}\right]\left(\mathrm{NaL}_{\mathrm{OiPr}}\right)$ were prepared 81 according to literature procedures. ${ }^{15}$

$82\left[D y\left(L_{O E t}\right)_{2}\right]\left(P F_{6}\right) \quad(1) . \mathrm{NaL}_{\mathrm{OEt}}(55.7 \mathrm{mg}, 0.10 \mathrm{mmol})$ and $83 \mathrm{NH}_{4} \mathrm{PF}_{6}(16.3 \mathrm{mg}, 0.10 \mathrm{mmol})$ were dissolved in water $(15 \mathrm{~mL})$ and 84 stirred for $10 \mathrm{~min}$. A yellow precipitate was generated immediately 85 upon the addition of $\mathrm{Dy}\left(\mathrm{NO}_{3}\right)_{3} \cdot 5 \mathrm{H}_{2} \mathrm{O}(17.4 \mathrm{mg}, 0.05 \mathrm{mmol})$ in water $86(2 \mathrm{~mL})$ to the ligand solution. The mixture was stirred for $12 \mathrm{~h}$, and 87 the precipitate was filtered and washed with water. Vapor diffusion of 88 diethyl ether into a methanol solution yielded crystals suitable for X89 ray analysis. Yield: $52.1 \mathrm{mg}(75.6 \%)$. Anal. Calcd for $90 \mathrm{C}_{34} \mathrm{H}_{70} \mathrm{Co}_{2} \mathrm{DyF}_{6} \mathrm{O}_{18} \mathrm{P}_{7}: \mathrm{C}, 29.62 ; \mathrm{H}, 5.12$. Found: C, 29.62; H, 5.04.

$91\left[Y\left(L_{\mathrm{OEt}}\right)_{2}\right]\left(\mathrm{PF}_{6}\right)$. The yttrium analogue was obtained by the same 92 procedure as that for compound 1, except that $\mathrm{Y}\left(\mathrm{NO}_{3}\right)_{3} \cdot 6 \mathrm{H}_{2} \mathrm{O}$ was 93 used instead of dysprosium(III). Yield: $72.3 \%$. Anal. Calcd for $94 \mathrm{C}_{34} \mathrm{H}_{70} \mathrm{Co}_{2} \mathrm{YF}_{6} \mathrm{O}_{18} \mathrm{P}_{7}$ : C, 31.30; H, 5.41. Found: C, 31.38; H, 5.49.

$95\left[\mathrm{Dy}\left(\mathrm{L}_{\mathrm{OEt}}\right)_{2}\right]\left(P F_{6}\right)$ Diluted 20-fold with $\left[\mathrm{Y}\left(\mathrm{L}_{\mathrm{OEt}}\right)_{2}\right]\left(\mathrm{PF}_{6}\right)$ (diluted-1). An 96 aqueous solution $(2 \mathrm{~mL})$ of $\mathrm{Dy}\left(\mathrm{NO}_{3}\right)_{3} \cdot 5 \mathrm{H}_{2} \mathrm{O}(0.005 \mathrm{mmol})$ and $97 \mathrm{Y}\left(\mathrm{NO}_{3}\right)_{3} \cdot 6 \mathrm{H}_{2} \mathrm{O}(0.10 \mathrm{mmol})$ was added to a solution of $\mathrm{NaL}_{\mathrm{OEt}}$ $98(111.4 \mathrm{mg}, 0.20 \mathrm{mmol})$ and $\mathrm{NH}_{4} \mathrm{PF}_{6}(32.6 \mathrm{mg}, 0.20 \mathrm{mmol})$ in water $99(15 \mathrm{~mL})$ with stirring. A yellow precipitate was generated, and the 100 mixture was stirred at room temperature for $12 \mathrm{~h}$. The precipitate was 101 filtered, washed with water, and dried in air. Anal. Calcd for $102 \mathrm{C}_{714} \mathrm{H}_{1470} \mathrm{Co}_{42} \mathrm{Y}_{20} \mathrm{DyF}_{126} \mathrm{O}_{378} \mathrm{P}_{147}: \mathrm{C}, 31.22 ; \mathrm{H}$, 5.39. Found: $\mathrm{C}$, 103 31.33; H, 5.35.

$104\left[D y\left(L_{\mathrm{OiPr}_{\mathrm{r}}}\right)_{2}\right]\left(P F_{6}\right)$ (2). Compound 2 was obtained by the same 105 procedure as that for compound $\mathbf{1}$ except for using $\mathrm{NaL}_{\mathrm{OiPr}}$ instead of $106 \mathrm{NaL}_{\mathrm{OEt}}$. Yield: 52.7\%. Anal. Calcd for $\mathrm{C}_{46} \mathrm{H}_{94} \mathrm{Co}_{2} \mathrm{DyF}_{6} \mathrm{O}_{18} \mathrm{P}_{7}$ : C, 35.77; 107 H, 6.00. Found: C, 35.77; H 6.08 .

108 Physical Measurements. Elemental analyses for carbon, hydro109 gen, and nitrogen were performed at the Elemental Analysis Service 110 Center of Sogang University. Powder X-ray diffraction data were 111 recorded using $\mathrm{Cu} \mathrm{K \alpha}(\lambda=1.5406 \AA)$ on a Rigaku Ultima III 112 diffractometer with a scan speed of $2^{\circ} / \mathrm{min}$ and a step size of $0.02^{\circ}$. 113 Magnetic susceptibilities for complexes $\mathbf{1}$ and $\mathbf{2}$ were carried out using 114 a Quantum Design SQUID susceptometer (direct current) and a 115 PPMS magnetometer (alternating current, ac). Diamagnetic correc116 tions of all samples were estimated from Pascal's tables.
Crystallographic Structure Determination. X-ray data for 1117 and 2 were collected on a Bruker SMART APEXII diffractometer 118 equipped with graphite-monochromated Mo $\mathrm{K} \alpha$ radiation $(\lambda=119$ $0.71073 \AA$ ) . A preliminary orientation matrix and cell parameters were 120 determined from three sets of $\phi$ scans at different starting angles. Data 121 frames were obtained at scan intervals of $0.5^{\circ}$ with an exposure time of 122 $10 \mathrm{~s} /$ frame. The reflection data were corrected for Lorentz and 123 polarization factors. Absorption corrections were carried out using 124 $S A D A B S$. The structures were solved by direct methods and refined by 125 full-matrix least-squares analysis using anisotropic thermal parameters 126 for non-hydrogen atoms with the SHELXTL program. In complex 2, 127 the $\mathrm{Cp}$ ring and diisopropylphosphito groups were disordered over 128 two sites ( 0.61 and 0.39 for parts $\mathrm{A}$ and $\mathrm{B}$, respectively) using PART, 129 and the isopropoxy groups were isotropically refined. All hydrogen 130 atoms were calculated at idealized positions and refined with the riding 131 models. The crystal data for $\mathbf{1}$ and $\mathbf{2}$ are summarized in Table S1. 132

Computational Details. The electronic structures of $\mathbf{1}$ and $\mathbf{2}$ were 133 determined using the CONDON computational package. ${ }^{16}$ As the 134 starting point, we used the crystal-field parameters (CFPs) determined 135 by the REC model $^{17}$ using the SIMPRE computational package ${ }^{18}$ and 136 assuming an ideal $D_{3 d}$ coordination environment with the $C_{3}$ axis 137 oriented in the $z$ direction. Then, the magnetic properties of $\mathbf{1}$ were 138 fitted using the full Hamiltonian. The resulting CFPs were tested with 139 the prediction of the experimental single-crystal magnetic anisotropy 140 measurements. Because of the chemical and structural similarity of 2, 141 the set of CFPs determined for $\mathbf{1}$ were used as input to fit the magnetic 142 properties of 2 . The free-ion parameters of dysprosium introduced in 143 CONDON (electron repulsion parameters $F^{k}(k=2,4,6)$ and spin- 144 orbit coupling constant $\xi_{\mathrm{SO}}$ ) were not varied during the fitting 145 procedures.

\section{RESULTS AND DISCUSSION}

Description of the Structures. To design the complexes, 148 we chose the tripodal ligands $\left[\mathrm{CpCo}\left[\mathrm{P}(\mathrm{O})(\mathrm{OR})_{2}\right]_{3}\right]^{-}$(Figure 149 S1), here shortened to $\mathrm{L}_{\mathrm{OR}}{ }^{-}(\mathrm{R}=\mathrm{Et}, i \mathrm{Pr})$, because huge steric 150 encumbrance is fundamental to hindering the tendency of rare 151 earths to move to higher coordination numbers (synthesis in 152 the experimental section). Both $\left[\mathrm{Dy}\left(\mathrm{L}_{\mathrm{OR}}\right)_{2}\right]^{+}$complexes 153 crystallize in the monoclinic space group $\mathrm{C} 2 / c$, with a $\mathrm{PF}_{6}^{-}{ }^{-} 54$ anion (Figure 1).

The dysprosium atom is sandwiched between six oxygen 156 atoms from two $\mathrm{L}_{\mathrm{OR}}{ }^{-}$ligands, successfully forming the planned 157 hexacoordinated structure. The Dy-O bond lengths are very 158 homogeneous, ranging between 2.249 and $2.257 \AA$ for $\mathbf{1}$ and 159 between 2.246 and $2.265 \AA$ for 2 . The angular deviation 160 parameter, defined as the sum of the deviation from $90^{\circ}$ of the 161 12 cis angles in the coordination sphere, is estimated to be $\Sigma=162$ $90.9^{\circ}$ for $\mathbf{1}$ and $71.6^{\circ}$ for $2 .^{19}$ The shape-deviation parameters 163 $S_{X}$ against an ideal octahedron $(X=\mathrm{O})$ and a trigonal prism $(X 164$ $=\mathrm{P})$ provide $S_{\mathrm{O}}=1.66$ and $S_{\mathrm{P}}=8.95$ for $\mathbf{1}$ and $S_{\mathrm{O}}=0.52$ and 165 $S_{\mathrm{p}}=15.11$ for 2 , showing that continuous-shape measures ${ }^{20} 166$ clearly indicate an octahedral geometry. While a perfect 167 octahedron is obtained in 2 , the use of different $\mathrm{L}_{\mathrm{OR}}{ }^{-}$ligands 168 allows distortion of the octahedron, producing a twisting angle 169 in 1 (Figure $1 \mathrm{~b}, \mathrm{~d})$. The diamagnetic $\mathrm{CpCo}$ and $\mathrm{PF}_{6}^{-}$parts are 170 positioned among the molecules and magnetically shield them, 171 where the shortest Dy-Dy distance is $12.1 \AA$ for $\mathbf{1}$ and $12.7 \AA 172$ for 2 (Figures S2 and S3).

Magnetic Properties. The magnetic susceptibilities $\chi_{\mathrm{m}}$ of 1174 and 2 (i.e., the ratio $M / H$ between the magnetization $M$ and 175 the magnetic field $H$ ) were measured at $H=1$ kOe down to 2.0176 $\mathrm{K}$ (Figures S5 and S6). The $\chi_{\mathrm{m}} \mathrm{T}$ values at room temperature 177 for $\mathbf{1}$ and $\mathbf{2}$ are 13.5 and $13.7 \mathrm{~cm}^{3} \mathrm{~K} \mathrm{~mol}^{-1}$, respectively, close to 178 the $14.2 \mathrm{~cm}^{3} \mathrm{~K} \mathrm{~mol}^{-1}$ value expected for the ${ }^{6} \mathrm{H}_{15 / 2}$ state of 179 dysprosium(III). $\chi_{\mathrm{m}} T$ first decreases gradually with $T$ and 180 plummets abruptly below $40 \mathrm{~K}$ because of depopulation of the 181 

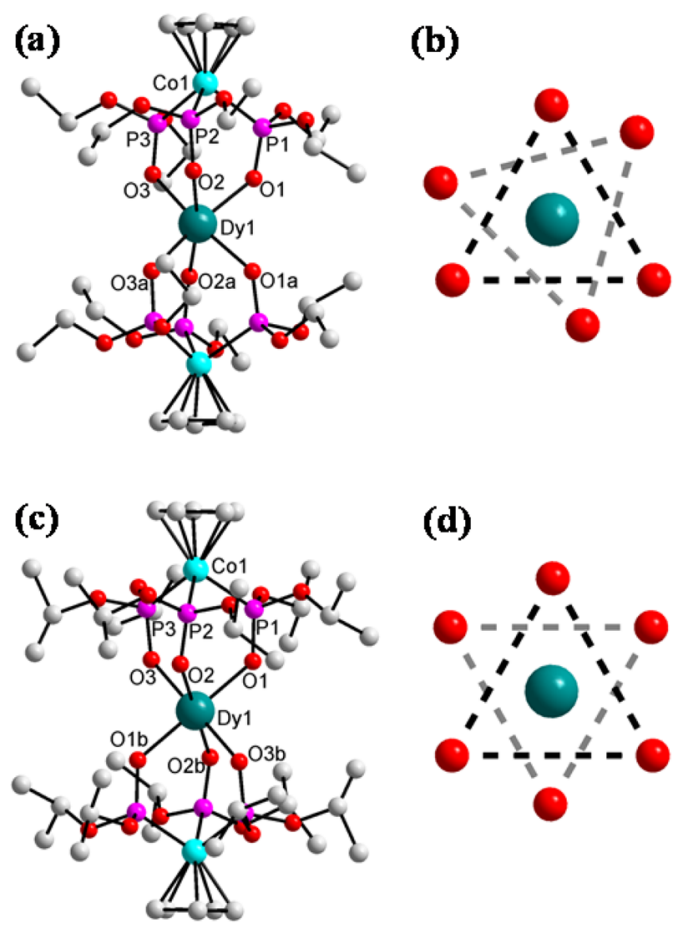

(d)

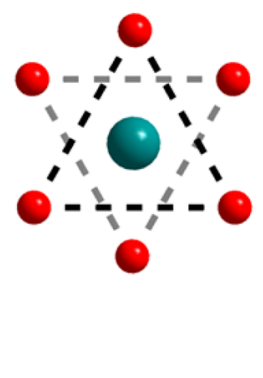

Figure 1. View of the crystal structure of (a) the cationic part of 1, (b) its dysprosium coordination environment, (c) the cationic part of $\mathbf{2}$, and (d) its dysprosium coordination environment. Hydrogen atoms are omitted for clarity.

182 Stark sublevels. ${ }^{21} M$ versus $H$ (Figures S7 and S8) likewise 183 indicates the presence of strong magnetic anisotropy. ${ }^{22}$

184 The magnetization dynamics were investigated by measuring 185 the in-phase $\chi_{\mathrm{m}}{ }^{\prime}$ and out-of-phase $\chi_{\mathrm{m}}{ }^{\prime \prime}$ responses of the samples 186 under a small field oscillating at frequency $\omega$ (Figures 2 and 187 S9-23). At $H=0$, quantum tunneling between degenerate 188 levels leads to very fast relaxation. ${ }^{23}$ With increasing $H$, the

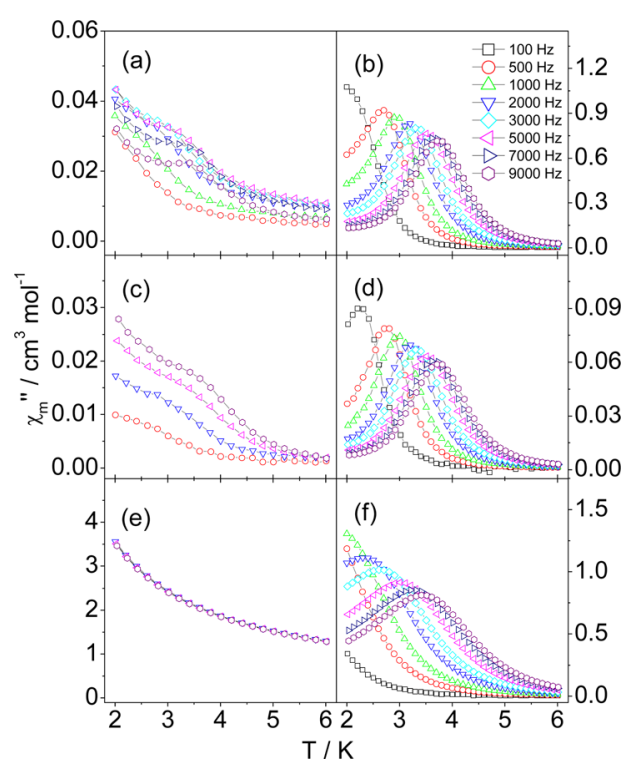

Figure 2. Temperature dependence of the out-of-phase component of the ac magnetic susceptibility, $\chi_{\mathrm{m}}{ }^{\prime \prime}$, in zero external field for (a) $\mathbf{1}$, (c) diluted-1, and (e) 2 and in the external field $H_{\mathrm{p}}$ where the relaxation is slowest for (b) 1, $H_{\mathrm{P}}=600 \mathrm{Oe}$, (d) diluted-1, $H_{\mathrm{P}}=450 \mathrm{Oe}$, and (f) 2, $H_{\mathrm{P}}=750$ Oe. relaxation time $\tau$ becomes slower, reaches a peak (at $H_{\mathrm{p}}=600189$ and 750 Oe for 1 and 2), and then decreases rapidly, as 190 expected for the progressive suppression of quantum tunneling 191 by $H$. Both 1 and 2 show a frequency-dependent peak in $\chi_{\mathrm{m}}{ }^{\prime \prime} 192$ versus $T$, and $\tau$ was extracted by fitting the ac curves with a 193 Debye model. The resulting Arrhenius plot shows a linear 194 region at high $T$ and a marked kink at lower $T$, indicating the 195 presence of a thermally activated mechanism still competing 196 with quantum tunneling.

In order to avoid overparameterization, we fit the ac 198 magnetometry data considering either Raman together with 199 quantum-tunneling processes (Figure S24) or a simple Orbach- 200 only process (Arrhenius plot), as expressed by the following 201 equation: ${ }^{24}$

$$
\tau=\tau_{0} \exp \left(U_{\text {eff }} / k T\right)
$$

The fit resulted in $\tau_{0}=2.4(5) \times 10^{-8} \mathrm{~s}$ and $\Delta E=18(2) \mathrm{cm}^{-1} 203$ for 1 and $\tau_{0}=6.4(5) \times 10^{-7} \mathrm{~s}$ and $\Delta E=9(1) \mathrm{cm}^{-1}$ for 2204 (Figure 3 ). Both $\tau_{0}$ values are compatible with SMM behavior, $205 \mathrm{f} 3$

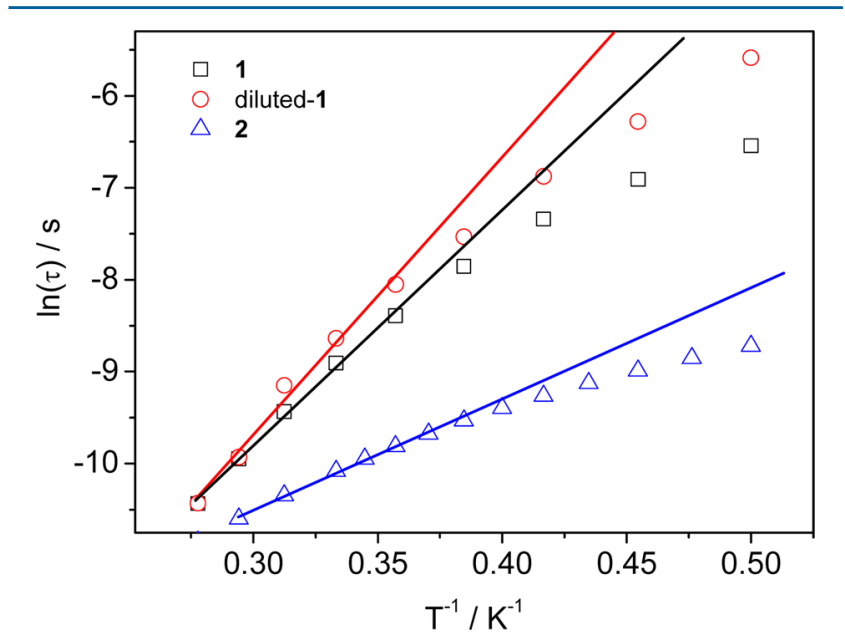

Figure 3. Dynamics of the magnetization as extracted from ac susceptibility measurements for $\mathbf{1}$ (black squares), 2 (blue triangles), and $\mathbf{1}$ diluted in a diamagnetic yttrium(III) matrix (red circles). The solid lines represent fits from the Arrhenius formula. For a Raman fit of 1 , see Figure S24.

and fitting of the Argand plots (Figures S13 and S18) shows a 206 narrow distribution of relaxation times, with the spreading 207 parameter $\alpha=0.2$ and 0.1 for 1 and 2, respectively. Doping 208 with yttrium(III) produces a very similar dynamic behavior, 209 with a slight decrease of $H_{\mathrm{p}}$ to $450 \mathrm{Oe}$, and longer $\tau$ values. The 210 resulting Arrhenius fit provides $\tau_{0}=6.0(6) \times 10^{-9} \mathrm{~s}$ and $\Delta E=211$ $21(2) \mathrm{cm}^{-1}$. The spin-flip attempt rate roughly matches the 212 expected value for spin-phonon processes and a barrier at the 213 limits of the extraction errors.

As seen in Figure 3, a simple Arrhenius plot does not capture 215 all of the physics in this system. Raman processes seem to be 216 dominant in the case of $\mathbf{1}$, where a satisfactory fit can be 217 obtained for $n=9$ (Figure S24). The $n$ values of $\mathbf{1}$ and diluted-1 218 are close to 9, consistent with the parameters obtained from the 219 fitting with an additional Orbach process. However, the Raman 220 model fails for $\mathbf{2}$. When $n$ is fixed to 9 , the fit of $\mathbf{2}$ is poor. 221

Single-Ion Anisotropy. We now examine the role of the 222 pseudoaxial local environment of the dysprosium(III) octahe- 223 dron in the anisotropy (Figures S25-S30). Because, in 1, the 224 dysprosium center is located on a $C_{2}$ rotation axis of the $C 2 / c 225$ space group, the molecular susceptibility tensor $\chi$ must coincide 226 
227 with the crystal one, and it is possible to obtain full magnetic 228 anisotropy by rotating around three orthogonal axes in the low$229 \mathrm{H}$ limit (Figure 4a). By a fitting and diagonalization procedure,

(a)

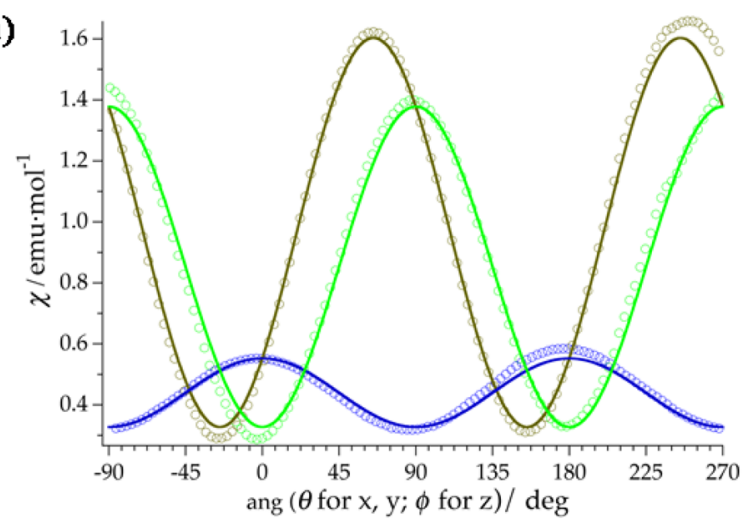

(b)

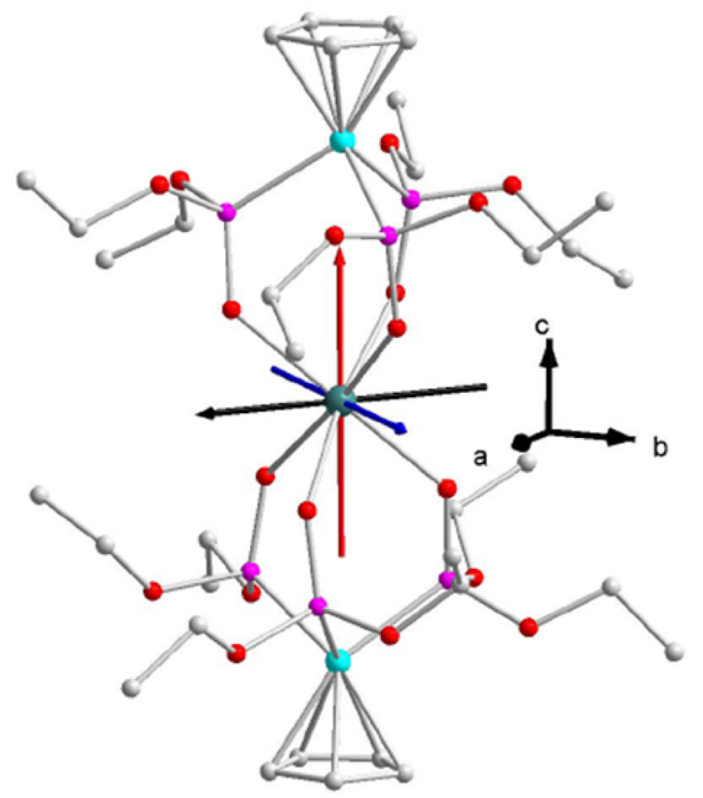

Figure 4. (a) Angular dependence of the magnetic susceptibility of 1 at $11 \mathrm{~K}$. The solid lines are the results of theoretical calculations. (b) Molecular structure showing the easy-axis direction determined from the experiment for an easy axis (red), a medium axis (blue), and a hard axis (black)

230 we obtain the orientation and magnitude of the principal axes at 231 different $T$ values. One eigenvalue is always dominant, with 232 very prominent easy-axis anisotropy oriented along the pseudo${ }_{233} \mathrm{C}_{3}$ molecular axis $(\mathrm{Co}-\mathrm{Co}$ direction; Figure $4 \mathrm{~b})$. This reveals a 234 clean example of uniaxial behavior, consistent with expectations 235 from the theory of octahedral transition-metal ions and without 236 the problems associated with higher-coordination systems. For 2372 , the same procedure is not possible because the crystal 238 symmetry prevents direct extraction of the molecular 239 susceptibility.

240 Theoretical Calculations. The crystallographic coordinates 241 of the first coordination sphere of $\mathbf{1}$ were idealized in order to 242 reduce the number of non-negligible CFPs. The molecule was 243 oriented with the pseudo- $C_{3}$ main axis pointing in the $z$ 244 direction. The idealization of the coordinates resulted in two 245 alternating equilateral triangles of point charges equidistant 246 with respect to the dysprosium ion $(\sim 2.25 \AA)$. An initial 247 individual fit of the powder magnetic susceptibility data using the REC model was performed, obtaining the following initial 248 set of CFPs in Wybourne notation $\left(\mathrm{cm}^{-1}\right): B_{20}=415 ; B_{40}=249$ -2066; $B_{43}=2070 ; B_{60}=432 ; B_{63}=2060 ; B_{66}=702$. They 250 were entered as input in the CONDON package, and only two 251 iterations were needed to obtain a very satisfactory fit $(\mathrm{SQX}=252$ $0.81 \%$; Figure S5), resulting in the following set of CFPs in 253 Wybourne notation $\left(\mathrm{cm}^{-1}\right): B_{20}=559(84) ; B_{40}=-3300(283) ; 254$ $B_{43}=1693(51) ; B_{60}=450(73) ; B_{63}=2034(96) ; B_{66}=255$ 718 (342). Magnetization at 4, 5, and $6 \mathrm{~K}$ was also reproduced 256 (Figure S7). According to this description, the first excited 257 Kramers doublet is found at $32 \mathrm{~cm}^{-1}$, and the total crystal-field 258 splitting of the ground-state $J$ is about $950 \mathrm{~cm}^{-1}$. The energy- 259 level distribution of the ground-state $J$ and the corresponding 260 wave functions are reported in Table S3. The lowest-energy 261 doublet from the next multiplet is located $2668 \mathrm{~cm}^{-1}$ above in 262 energy, placed at $\sim 3618 \mathrm{~cm}^{-1}$. The ground-state Kramers 263 doublet in the easy-axis orientation is described by $89.6 \%$ of | 264 $\left.\pm^{11} / 2\right\rangle$ with a $9.4 \%$ contribution of $| \pm 5 / 2\rangle$, which corresponds 265 to an effective $g_{\|}$of $13.74\left[g_{\|}(\exp )=13.83\right]$ and $g_{\perp}=0.48$. This 266 description is almost in perfect agreement with the observed 267 easy-axis behavior (Figure S29). Thus, the angular dependence 268 of the susceptibility of $\mathbf{1}$ is also reproduced accurately (Figures 269 S31-S33), validating once more the theoretical results. Also, 270 because of the chemical similarity and similar coordination 271 environment of 2, we have used the calculated set of CFPs as 272 the starting point to model the magnetic susceptibility of $\mathbf{2}$. An 273 excellent agreement with SQX $=0.69 \%$ (Figure S6) was 274 achieved with the following set of CFPs in $\mathrm{cm}^{-1}: B_{20}=275$ 467(301); $B_{40}=-3605(990) ; B_{43}=596(215) ; B_{60}=164(133) ; 276$ $B_{63}=1545(281) ; \quad B_{66}=-357(2489)$, reproducing also 277 magnetization curves (Figure S8). The uncertainties estimated 278 for the CFPs are reported in Table S2. The large uncertainties 279 found in some of the CFPs likely stem from the sole 280 consideration of the powder magnetic susceptibility data; i.e., 281 single-crystal data were not taken into account. One should 282 consider these uncertainties as an upper limit because (1) we 283 introduced structural information as a starting point and (2) we 284 are predicting instead of fitting the magnetic anisotropy 285 experimental data. The energy-level scheme of the ground- 286 state multiplet and the microstate contributions to the wave 287 functions are also reported in Table S3. The first excited-state 288 doublet is placed at about $44 \mathrm{~cm}^{-1}$, and the total splitting is 289 $\sim 853 \mathrm{~cm}^{-1}$. In this case, the ground-state wave function is more 290 pure, being determined by $98.4 \%$ of $\left| \pm^{11} / 2\right\rangle$ according to our 291 calculations. This leads to a slightly larger value of $g_{\|}(14.54) 292$ compared with that of 1 (13.74). Nevertheless, this subtle 293 difference would need a finer determination by spectroscopic 294 measurements.

In square-antiprismatic systems, the dynamics depend on the 296 axial compression or elongation of the complex, and the 297 presence of a twist angle lowers the barrier and introduces 298 additional transverse terms that favor quantum tunneling. We 299 now use an electrostatic model to reproduce the observations 300 and study structure-property correlations. The states of rare 301 earths are, in general, a linear combination of pure $\left|m_{j}\right\rangle$ spin 302 sublevels, with mixing introduced by the high spin-orbit 303 coupling and the symmetry of the system. In ligand-field 304 theory, the orientation of the quantization axis depends only on 305 the distribution of the charges around the spin. By expanding 306 the electron density of the $\left|m_{j}\right\rangle$ states into a series of spherical 307 harmonics $Y_{k}^{0}$ (with $\left.k \leq 6\right)$, ${ }^{25}$ we can calculate the potential 308 energy of each pure $\left|m_{j}\right\rangle$ state as a function of the orientation, 309 so as to find the resulting preferred orientation of the easy axis. ${ }^{7} 310$ 
311 The results obtained considering the charges inside $\mathbf{1}$ and $\mathbf{2}$ are 312 plotted in Figure 5a,b against the azimuthal $\varphi$ and polar $\theta$

a)

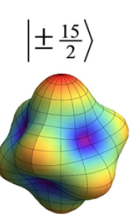

b)

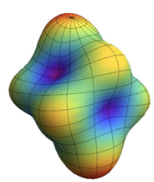

c)

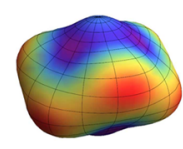

d)

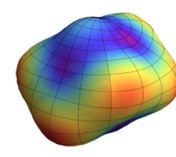

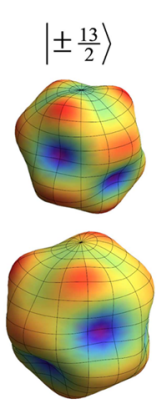
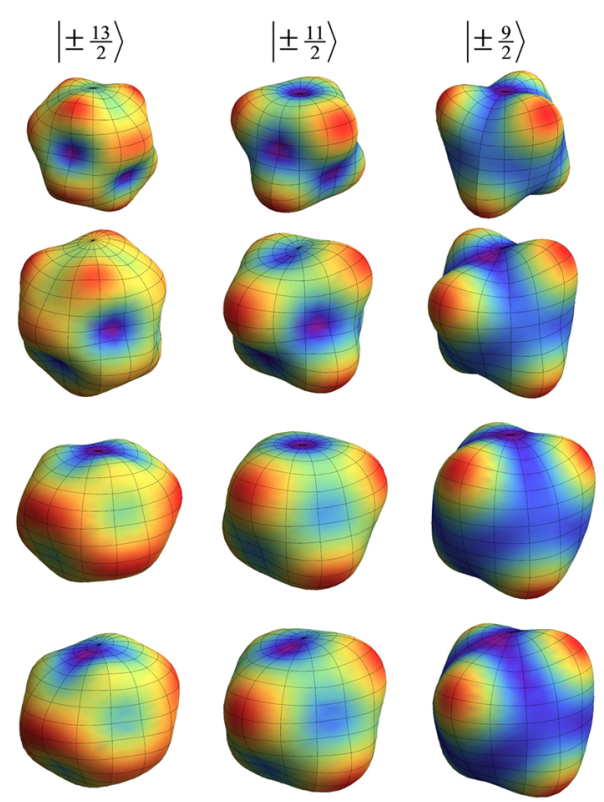

Figure 5. Potential energy surfaces, represented in spherical coordinates, for the different orientations of the pure $\left|m_{j}\right\rangle$ spin sublevels (only the highest four displayed) for (a) 1 and (b) 2. The same plots for 1 (c) and 2 (d) were obtained by removing the positively charged and diamagnetic cobalt(III) ions. The colorscale indicates the energy (violet, lowest; red, highest), and the vertical position is along the pseudo- $C_{3}$ axis.

313 angles of the spherical coordinates. From the results, it is 314 evident that, for 1 , both the $|11 / 2\rangle$ and $|9 / 2\rangle$ states have an 315 absolute energy minimum at $\theta=0$, which corresponds to the 316 direction of the pseudo- $C_{3}$ molecular axis. They must thus be 317 the dominant contribution to stabilize the observed orientation 318 of the magnetization easy axis. This is in perfect agreement with 319 the phenomenological fit by CONDON for both complexes. On 320 the contrary, the highest $m_{j}$ state, $\left|{ }^{15} / 2\right\rangle$, often assumed as the 321 dominant ground-state contribution in rare-earth SMMs, 322 stabilizes a tilted easy axis. A more regular octahedral geometry, 323 as observed in passing from $\mathbf{1}$ to 2 , changes the preferred easy324 axis orientation of the $|11 / 2\rangle$ contribution, moving it to a 325 transverse geometry and predictably lowering the barrier. 326 According to these calculations, the diamagnetic cobalt(III) 327 ions are revealed to be surprisingly important in $\mathbf{1}$ because they 328 provide a strong positive charge along the pseudo- $C_{3}$ axis. This 329 is fundamental in reducing the $\left.\mathrm{I}^{15} / 2\right\rangle$ contribution to the ground 330 state because the highest $m_{j}$ state is predicted to be 331 preferentially oriented where the negative charge is most 332 dense. Without the cobalt(III) ions, the coordinating atoms and $333 \mathrm{Cp}^{-}$rings would stabilize a $\left.\left.\right|^{15} / 2\right\rangle$ ground state (Figures $5 \mathrm{c}$, d 334 and S34 and S35).

\section{$335 \square$ CONCLUSIONS}

$336 \mathrm{We}$ have rationally used bulky tripodal ligands to obtain a 337 nearly ideal octahedral geometry around rare earths, resulting in 338 two new dysprosium(III) SMMs. We have subjected them to a 339 detailed experimental and theoretical magnetic analysis. When 340 the REC model (an effective point-charge approach) and the 341 CONDON software (using the full Hamiltonian) are 342 combined, it has been possible to theoretically simulate the magnetic anisotropy, including the susceptibility along three 343 perpendicular rotations. Furthermore, using these consider- 344 ations, we could rationalize the role of the different constituents 345 of the molecule, including the diamagnetic cobalt(III) ions, 346 which provide a strong positive charge along the pseudo- $C_{3} 347$ axis, making it the main magnetic axis in spite of the near- 348 octahedral coordination sphere.

\section{ASSOCIATED CONTENT}

\section{S Supporting Information}

The Supporting Information is available free of charge on the 352 ACS Publications website at DOI: 10.1021/acs.inorg- 353 chem.6b03118.

Additional structural and magnetic data for the 355 complexes (PDF)

X-ray crystallographic file in CIF format for 1 (CIF) 357

X-ray crystallographic file in CIF format for $\mathbf{2}$ (CIF) 358

\section{AUTHOR INFORMATION}

\section{Corresponding Authors}

*E-mail: alejandro.gaita@uv.es.

*E-mail: lapo.bogani@materials.ox.ac.uk.

*E-mail: cshong@korea.ac.kr.

ORCID $\odot$

ShangDa Jiang: 0000-0003-0204-9601

Alejandro Gaita-Ariño: 0000-0002-1600-8627

Chang Seop Hong: 0000-0002-4329-4745

\section{Author Contributions}

${ }^{\S}$ The manuscript was written through contributions of all 369 authors. All authors have given approval to the final version of 370 the manuscript. These authors contributed equally.

Notes

The authors declare no competing financial interest.

371

\section{ACKNOWLEDGMENTS}

We are thankful for financial support from the Korean Basic 375 Science Research Program (NRF-2015R1A2A1A10055658), an 376 KCRC grant (Grant NRF-2012-0008901), the Korean Priority 377 Research Centers Program (NRF2010-0020209), EU (ERC- 378 CoG-647301 DECRESIM, ERC-StG-338258 OptoQMol, and 379 COST Action 15128 MOLSPIN), the Spanish MINECO 380 (Grant MAT2014-56143-R, CTQ2014-52758-P and Excellence 381 Unit María de Maeztu MDM-2015-0538), and the Generalitat 382 Valenciana (Prometeo Programme of excellence). A.G.-A. 383 acknowledges funding by the MINECO (Ramón y Cajal 384 contract).

\section{REFERENCES}

386

(1) (a) Ishikawa, N.; Sugita, M.; Ishikawa, T.; Koshihara, S.-y.; Kaizu, 387 Y. Lanthanide Double-Decker Complexes Functioning as Magnets at 388 the Single-Molecular Level. J. Am. Chem. Soc. 2003, 125, 8694-8695. 389 (b) Ishikawa, N.; Sugita, M.; Ishikawa, T.; Koshihara, S.-y.; Kaizu, Y. 390 Mononuclear Lanthanide Complexes with a Long Magnetization 391 Relaxation Time at High Temperatures: A New Category of Magnets 392 at the Single-Molecular Level. J. Phys. Chem. B 2004, 108, 11265- 393 11271.

(2) (a) Woodruff, D. N.; Winpenny, R. E.; Layfield, R. A. Lanthanide 395 single-molecule magnets. Chem. Rev. 2013, 113 (7), 5110-5148. 396 (b) Liddle, S. T.; van Slageren, J. Improving f-element single molecule 397 magnets. Chem. Soc. Rev. 2015, 44 (19), 6655-6669. (c) Gregson, M.; 398 Chilton, N. F.; Ariciu, A.-M.; Tuna, F.; Crowe, I. F.; Lewis, W.; Blake, 399 A. J.; Collison, D.; McInnes, E. J. L.; Winpenny, R. E. P.; Liddle, S. T. 400 
401 A monometallic lanthanide bis(methanediide) single molecule magnet 402 with a large energy barrier and complex spin relaxation behavior. 403 Chem. Sci. 2016, 7 (1), 155-165. (d) Chen, Y. C.; Liu, J. L.; Ungur, L.; 404 Liu, J.; Li, Q. W.; Wang, L. F.; Ni, Z. P.; Chibotaru, L. F.; Chen, X. M.; 405 Tong, M. L. Symmetry-Supported Magnetic Blocking at $20 \mathrm{~K}$ in 406 Pentagonal Bipyramidal Dy(III) Single-Ion Magnets. J. Am. Chem. Soc. 407 2016, 138 (8), 2829-2837. (e) Liu, J.; Chen, Y. C.; Liu, J. L.; Vieru, 408 V.; Ungur, L.; Jia, J. H.; Chibotaru, L. F.; Lan, Y.; Wernsdorfer, W.; 409 Gao, S.; Chen, X. M.; Tong, M. L. A Stable Pentagonal Bipyramidal 410 Dy(III) Single-Ion Magnet with a Record Magnetization Reversal 411 Barrier over 1000 K. J. Am. Chem. Soc. 2016, 138 (16), 5441-5450. 412 (f) Coutinho, J. T.; Antunes, M. A.; Pereira, L. C.; Bolvin, H.; Marcalo, 413 J.; Mazzanti, M.; Almeida, M. Single-ion magnet behavior in $414\left[\mathrm{U}\left(\mathrm{Tp}\left(\mathrm{Me}_{2}\right)\right)_{2} \mathrm{I}\right]$. Dalton Trans. 2012, 41 (44), 13568-13571.

415 (3) (a) Martínez-Pérez, M. J.; Cardona-Serra, S.; Schlegel, C.; Moro, 416 F.; Alonso, P. J.; Prima-García, H.; Clemente-Juan, J. M.; Evangelisti, 417 M.; Gaita-Arino, A.; Sesé, J.; van Slageren, J.; Coronado, E.; Luis, F. 418 Gd-based single-ion magnets with tunable magnetic anisotropy: 419 molecular design of spin qubits. Phys. Rev. Lett. 2012, 108 (24), 420 247213. (b) Luis, F.; Repollés, A.; Martínez-Pérez, M. J.; Aguilà, D.; 421 Roubeau, O.; Zueco, D.; Alonso, P. J.; Evangelisti, M.; Camón, A.; 422 Sesé, J.; Barrios, L. A.; Aromí, G. Molecular prototypes for spin-based 423 CNOT and SWAP quantum gates. Phys. Rev. Lett. 2011, 107 (11), 424 117203. (c) Baldoví, J. J.; Cardona-Serra, S.; Clemente-Juan, J. M.; 425 Coronado, E.; Gaita-Ariño, A.; Prima-Garcia, H. Coherent manipu426 lation of spin qubits based on polyoxometalates: the case of the single 427 ion magnet $\left[\mathrm{GdW}_{30} \mathrm{P}_{5} \mathrm{O}_{110}\right]_{14}$. Chem. Commun. 2013, 49 (79), 8922428 8924. (d) Baldoví, J. J.; Cardona-Serra, S.; Clemente-Juan, J. M.; 429 Coronado, E.; Gaita-Ariño, A.; Palii, A. Rational design of single-ion 430 magnets and spin qubits based on mononuclear lanthanoid complexes. 431 Inorg. Chem. 2012, 51 (22), 12565-12574. (e) Thiele, S.; Balestro, F.; 432 Ballou, R.; Klyatskaya, S.; Ruben, M.; Wernsdorfer, W. Electrically 433 driven nuclear spin resonance in single-molecule magnets. Science 434 2014, 344 (6188), 1135-1138.

435 (4) (a) Ding, Y.-S.; Chilton, N. F.; Winpenny, R. E. P.; Zheng, Y.-Z. 436 On Approaching the Limit of Molecular Magnetic Anisotropy:A Near437 Perfect Pentagonal Bipyramidal Dysprosium(III)Single-Molecule 438 Magnet. Angew. Chem., Int. Ed. 2016, 55, 16071-16074. (b) Zadrozny, 439 J. M.; Niklas, J.; Poluektov, O. G.; Freedman, D. E. Millisecond 440 Coherence Time in a Tunable Molecular Electronic Spin Qubit. ACS 441 Cent. Sci. 2015, 1 (9), 488-492.

442 (5) (a) Atzori, M.; Morra, E.; Tesi, L.; Albino, A.; Chiesa, M.; Sorace, 443 L.; Sessoli, R. Quantum Coherence Times Enhancement in 444 Vanadium(IV)-based Potential Molecular Qubits: the Key Role of 445 the Vanadyl Moiety. J. Am. Chem. Soc. 2016, 138 (35), 11234-11244. 446 (b) Lunghi, A.; Totti, F.; Sessoli, R; Sanvito, S. The role of 447 anharmonic phonons in under-barrier spin relaxation of single 448 molecule magnets. Nat. Commun. 2017, 8, 14620. (c) Escalera449 Moreno, L.; Suaud, N.; Gaita-Ariño, A.; Coronado, E. Determining 450 Key Local Vibrations in the Relaxation of Molecular Spin Qubits and 451 Single Molecule Magnets. J. Phys. Chem. Lett. 2017, 8, 1695.

452 (6) (a) Clemente-Juan, J. M.; Coronado, E.; Gaita-Ariño, A. 453 Magnetic polyoxometalates: from molecular magnetism to molecular 454 spintronics and quantum computing. Chem. Soc. Rev. 2012, 41 (22), 455 7464-7478. (b) Cardona-Serra, S.; Clemente-Juan, J. M.; Coronado, 456 E.; Gaita-Ariño, A.; Camón, A.; Evangelisti, M.; Luis, F.; Martínez457 Peréz, M. J.; Sesé, J. Lanthanoid single-ion magnets based on 458 polyoxometalates with a 5 -fold symmetry: the series $\left[\mathrm{LnP}_{5} \mathrm{~W}_{30} \mathrm{O}_{110}\right]^{12-}$ 459 (Ln3+ = Tb, Dy, Ho, Er, Tm, and Yb). J. Am. Chem. Soc. 2012, 134 460 (36), 14982-14990.

461 (7) Chilton, N. F.; Collison, D.; McInnes, E. J.; Winpenny, R. E.; 462 Soncini, A. An electrostatic model for the determination of magnetic 463 anisotropy in dysprosium complexes. Nat. Commun. 2013, 4, 2551. 464 (8) (a) Zhang, P.; Guo, Y.-N.; Tang, J. Recent advances in 465 dysprosium-based single molecule magnets: Structural overview and 466 synthetic strategies. Coord. Chem. Rev. 2013, 257 (11-12), 1728467 1763. (b) Qian, K.; Baldoví, J. J.; Jiang, S.-D.; Gaita-Ariño, A.; Zhang, 468 Y.-Q.; Overgaard, J.; Wang, B.-W.; Coronado, E.; Gao, S. Does the thermal evolution of molecular structures critically affect the magnetic 469 anisotropy? Chem. Sci. 2015, 6 (8), 4587-4593.

(9) (a) Jiang, S. D.; Wang, B. W.; Su, G.; Wang, Z. M.; Gao, S. A 471 mononuclear dysprosium complex featuring single-molecule-magnet 472 behavior. Angew. Chem., Int. Ed. 2010, 49 (41), 7448-7451. (b) Bi, Y.; 473 Guo, Y. N.; Zhao, L.; Guo, Y.; Lin, S. Y.; Jiang, S. D.; Tang, J.; Wang, 474 B. W.; Gao, S. Capping ligand perturbed slow magnetic relaxation in 475 dysprosium single-ion magnets. Chem. - Eur. J. 2011, 17 (44), 12476- 476 124781. (c) Li, D. P.; Wang, T. W.; Li, C. H.; Liu, D. S.; Li, Y. Z.; You, 477 X. Z. Single-ion magnets based on mononuclear lanthanide complexes 478 with chiral Schiff base ligands $\left[\mathrm{Ln}(\mathrm{FTA})_{3} \mathrm{~L}\right](\mathrm{Ln}=\mathrm{Sm}, \mathrm{Eu}, \mathrm{Gd}, \mathrm{Tb}$ and 479 Dy). Chem. Commun. 2010, 46 (17), 2929-2931. (d) Wang, H.; 480 Wang, K.; Tao, J.; Jiang, J. Twist angle perturbation on mixed 481 (phthalocyaninato)(porphyrinato) dysprosium(III) double-decker 482 SMMs. Chem. Commun. 2012, 48 (24), 2973-2975. (e) Chen, G. J.; 483 Guo, Y. N.; Tian, J. L.; Tang, J.; Gu, W.; Liu, X.; Yan, S. P.; Cheng, P.; 484 Liao, D. Z. Enhancing anisotropy barriers of dysprosium(III) single- 485 ion magnets. Chem. - Eur. J. 2012, 18 (9), 2484-2487.

(10) Baldoví, J. J.; Rosaleny, L. E.; Ramachandran, V.; Christian, J.; 487 Dalal, N. S.; Clemente-Juan, J. M.; Yang, P.; Kortz, U.; Gaita-Ariño, A.; 488 Coronado, E. Molecular spin qubits based on lanthanide ions 489 encapsulated in cubic polyoxopalladates: design criteria to enhance 490 quantum coherence. Inorg. Chem. Front. 2015, 2, 893-897.

491

(11) Shiddiq, M.; Komijani, D.; Duan, Y.; Gaita-Ariño, A.; Coronado, 492 E.; Hill, S. Enhancing coherence in molecular spin qubits via atomic 493 cloc transitions. Nature 2016, 531, 348-351.

(12) Jenkins, M. D.; Duan, Y.; Diosdado, B.; García-Ripoll, J. H.; 495 Gaita-Ariño, A.; Giménez-Saiz, C.; Alonso, P. J.; Coronado, E.; Luis, F. 496 Coherent manipulation of three-quit states in a molecular single-ion 497 magnet. Phys. Rev. B: Condens. Matter Mater. Phys. 2017, 95, 064423. 498 (13) Liu, J.-L.; Chen, Y.-C.; Zheng, Y.-Z.; Lin, W.-Q.; Ungur, L.; 499 Wernsdorfer, W.; Chibotaru, L. F.; Tong, M.-L. Switching the 500 anisotropy barrier of a single-ion magnet by symmetry change from 501 quasi- $\mathrm{D}_{5 \mathrm{~h}}$ to quasi- $\mathrm{O}_{\mathrm{h}}$. Chem. Sci. 2013, 4, 3310-3316. 502

(14) (a) Zeng, D.; Ren, M.; Bao, S.-S.; Li, L.; Zheng, L.-M. A 503 luminescent heptanuclear DyIr 6 complex showing field-induced slow 504 magnetization relaxation. Chem. Commun. 2014, 50, 8356-8359. 505 (b) König, S. N.; Chilton, N. F.; Maichle-Mössmer, C.; Pineda, E. M.; 506 Pugh, T.; Anwander, R.; Layfield, R. A. Fast magnetic relaxation in an 507 octahedral dysprosium tetramethyl-aluminate complex. Dalton Trans. 508 2014, 43, 3035-3038. (c) Klementyeva, S. V.; Afonin, M. Y.; 509 Bogomyakov, A. S.; Gamer, M. T.; Roesky, P. W.; Konchenko, S. K. 510 Mono- and Dinuclear Rare-Earth Chlorides Ligated by a Mesityl0Sub- 511 stituted $\beta$-Diketiminate. Eur. J. Inorg. Chem. 2016, 2016, 3666-3672. 512 (15) (a) Kläui, W.; Eberspach, W.; Gütlich, P. Spin-Crossover Cobalt 513 (III) Complexes: Steric and Electronic Control of Spin State. Inorg. 514 Chem. 1987, 26, 3977-3982. (b) Kläui, W.; Eberspach, W.; Schwarz, 515 R. ZUR REAKTIVITÄT VON COBALTOCEN UND NICKEL- 516 OCEN GEGENÜBER SEKUNDÄREN PHOSPHINCHALKOGE- 517 NIDEN: EIN WEG ZU MEHRZÄHNIZEN SAUERSTOFF-UND 518 SCHWEFEL-LIGANDEN. J. Organomet. Chem. 1983, 252, 347-357. 519 (16) (a) van Leusen, J.; Speldrich, M.; Schilder, H.; Kögerler, P. 520 Comprehensive insight into molecular magnetism via CONDON: Full 521 vs. effective models. Coord. Chem. Rev. 2015, 289-290, 137-148. 522 (b) Speldrich, M.; Schilder, H.; Lueken, H.; Kögerler, P. A 523 Computational Framework for Magnetic Polyoxometalates and 524 Molecular Spin Structures: CONDON 2.0. Isr. J. Chem. 2011, 51, 525 215-227.

526

(17) Baldoví, J. J.; Borrás-Almenar, J. J.; Clemente-Juan, J. M.; 527 Coronado, E.; Gaita-Ariño, A. Modeling the properties of lanthanoid 528 single-ion magnets using an effective point-charge approach. Dalton 529 Trans. 2012, 41 (44), 13705-13710.

(18) Baldoví, J. J.; Cardona-Serra, S.; Clemente-Juan, J. M.; 531 Coronado, E.; Gaita-Ariño, A.; Palii, A. SIMPRE: A software package 532 to calculate crystal field parameters, energy levels, and magnetic 533 properties on mononuclear lanthanoid complexes based on charge 534 distributions. J. Comput. Chem. 2013, 34 (22), 1961-1967. 
536 (19) Drew, M. G. B.; Harding, C. J.; McKee, V.; Morgan, G. G.; 537 Nelson, J. Geometric Control of Manganese Redox State. J. Chem. Soc., 538 Chem. Commun. 1995, 1035-1038.

539 (20) Alvarez, S.; Alemany, P.; Casanova, D.; Cirera, J.; Llunell, M.; 540 Avnir, D. Shape maps and polyhedral interconversion paths in 541 transition metal chemistry. Coord. Chem. Rev. 2005, 249 (17-18), $542 \quad 1693-1708$.

543 (21) (a) Sutter, J.-P.; Kahn, M. L.; Kahn, O. Conclusive 544 Demonstration of the Ferromagnetic Nature of the Interaction 545 Between Holmium(III) and Aminoxyl Radicals. Adv. Mater. 1999, 546 11, 863-865. (b) Kahn, M. L.; Sutter, J.-P.; Golhen, S.; Guionneau, P.; 547 Ouahab, L.; Kahn, O.; Chasseau, D. Systematic Investigation of the 548 Nature of The Coupling between a $\operatorname{Ln}($ III) Ion ( $\mathrm{Ln}) \mathrm{Ce}(\mathrm{III})$ to $549 \mathrm{Dy}(\mathrm{III}))$ and Its Aminoxyl Radical Ligands. Structural and Magnetic 550 Characteristics of a Series of $\left\{\operatorname{Ln}(\text { organic radical })_{2}\right\}$ Compounds and 551 the Related $\left\{\operatorname{Ln}(\text { Nitrone })_{2}\right\}$ Derivatives. J. Am. Chem. Soc. 2000, 122, $5523413-3421$.

553 (22) (a) Tang, J.; Hewitt, I.; Madhu, N. T.; Chastanet, G.; 554 Wernsdorfer, W.; Anson, C. E.; Benelli, C.; Sessoli, R.; Powell, A. K. 555 Dysprosium triangles showing single-molecule magnet behavior of 556 thermally excited spin states. Angew. Chem., Int. Ed. 2006, 45 (11), 557 1729-1733. (b) Layfield, R. A.; McDouall, J. J.; Sulway, S. A.; Tuna, 558 F.; Collison, D.; Winpenny, R. E. Influence of the N-bridging ligand on 559 magnetic relaxation in an organometallic dysprosium single-molecule 560 magnet. Chem. - Eur. J. 2010, 16 (15), 4442-4446. (c) Gamer, M. T.; 561 Lan, Y.; Roesky, P. W.; Powell, A. K.; Clérac, R. Pentanuclear 562 Dysprosium Hydroxy Cluster Showing Single-Molecule-Magnet 563 Behavior. Inorg. Chem. 2008, 47, 6581-6583. (d) Zheng, Y.-Z.; Lan, 564 Y.; Anson, C. E.; Powell, A. K. Anion-Perturbed Magnetic Slow 565 Relaxation in Planar $\left\{\mathrm{Dy}_{4}\right\}$ Clusters. Inorg. Chem. 2008, 47, 1081356610815.

567 (23) (a) Blagg, R. J.; Tuna, F.; McInnes, E. J.; Winpenny, R. E. 568 Pentametallic lanthanide-alkoxide square-based pyramids: high energy 569 barrier for thermal relaxation in a holmium single molecule magnet. 570 Chem. Commun. 2011, 47 (38), 10587-10589. (b) Koo, B. H.; Lim, K. 571 S.; Ryu, D. W.; Lee, W. R.; Koh, E. K.; Hong, C. S. A unique 572 tetranuclear $\mathrm{Er}(\mathrm{III})_{4}$ cluster exhibiting field-induced single-molecule 573 magnetism. Chem. Commun. 2012, 48 (19), 2519-2521. (c) Sorace, 574 L.; Benelli, C.; Gatteschi, D. Lanthanides in molecular magnetism: old 575 tools in a new field. Chem. Soc. Rev. 2011, 40 (6), 3092-3104. 576 (d) Jiang, S. D.; Wang, B. W.; Sun, H. L.; Wang, Z. M.; Gao, S. An 577 organometallic single-ion magnet. J. Am. Chem. Soc. 2011, 133 (13), 578 4730-4733. (e) Snyder, J.; Ueland, B. G.; Slusky, J. S.; Karunadasa, H.; 579 Cava, R. J.; Mizel, A.; Schiffer, P. Quantum-classical reentrant 580 relaxation crossover in $\mathrm{Dy}_{2} \mathrm{Ti}_{2} \mathrm{O}_{7}$ spin ice. Phys. Rev. Lett. 2003, 91 581 (10), 107201.

582 (24) (a) Brown, A. J.; Pinkowicz, D.; Saber, M. R.; Dunbar, K. R. A 583 Trigonal-Pyramidal Erbium(III) Single-Molecule Magnet. Angew. 584 Chem., Int. Ed. 2015, 54 (20), 5864-5868. (b) Meihaus, K. R.; 585 Minasian, S. G.; Lukens, W. W., Jr.; Kozimor, S. A.; Shuh, D. K.; 586 Tyliszczak, T.; Long, J. R. Influence of pyrazolate vs N-heterocyclic 587 carbene ligands on the slow magnetic relaxation of homoleptic 588 trischelate lanthanide(III) and uranium(III) complexes. J. Am. Chem. 589 Soc. 2014, 136 (16), 6056-6068.

590 (25) Sievers, J. Asphericity of 4f-Shells in Their Hund's Rule Ground 591 States. Z. Phys. B: Condens. Matter Quanta 1982, 45, 289-296. 